\title{
Typed Dynamic Control Operators for Delimited Continuations
}

\author{
Yukiyoshi Kameyama and Takuo Yonezawa \\ Department of Computer Science, University of Tsukuba \\ kameyama@acm.org, yone@logic.cs.tsukuba.ac.jp
}

\begin{abstract}
We study the dynamic control operators for delimited continuations, control and prompt. Based on recent developments on purely functional CPS translations for them, we introduce a polymorphically typed calculus for these control operators which allows answer-type modification. We show that our calculus enjoys type soundness and is compatible with the CPS translation. We also show that the typed dynamic control operators can macro-express the typed static ones (shift and reset), while the converse direction is not possible, which exhibits a sharp contrast with the type-free case.

Keywords: Type System, Delimited Continuation, Dynamic Control Operator, CPS Translation, Polymorphism, Expressivity.
\end{abstract}

\section{Introduction}

Delimited continuations represent not the rest of the computation as with traditional continuations [18], but only part of the rest of the computation. As such, delimited continuations have been used to model backtracking in contrast to traditional continuations that are used to model jumps.

In direct style, traditional continuations are accessed with control operators such as call/cc. There is, however, more variety for delimited continuations:

- Felleisen [12] proposed a control delimiter to signify part of an evaluation context, or a delimited continuation. This lead to the new control operators control and prompt, which are called dynamic control operators.

- Danvy and Filinski discovered that delimited continuations are supported by an already existing formalism of 2CPS, the image of iterated CPS translations. They proposed new control operators shift and reset in the directstyle counterpart [9]. They are called static control operators.

Since these proposals, the static control operators have been intensively studied while the dynamic ones are relatively less studied. For shift/reset, there are a number of theoretical results $[13,14]$ as well as useful examples in partial evaluation, one-pass CPS translation, and mobile codes, while we do not find many corresponding works for control/prompt in the literature, partly due to the difficulty in reasoning about the dynamic features of control/prompt.

Recently, several authors have started to obtain better understanding for dynamic ones, and to connect dynamic and static ones. Shan [7] macro-expressed 
control and prompt in terms of shift and reset using recursive types. (See also Kiselyov's work [15].) Biernacki, Danvy, and Millikin [6] derived a CPS translation for control and prompt from a definitional abstract machine, and gave another encoding of control/prompt by shift/reset. Dybvig, Peyton Jones, and Sabry [11] gave a uniform monadic framework for delimited continuations including control/prompt. However, no work has studied the direct-style type system for control/prompt in a way comparative to the type system for shift/reset. The proposed encodings were done in either type-free or recursively typed settings, ${ }^{1}$ and the proposed CPS translations assumed a restricted direct-style type system in that the control effect of "answer-type modification" was not allowed. We think this effect is indispensable for delimited continuations, for instance, it is needed to type the printf function in direct style $[8,1]$.

In this paper, we propose a direct-style type system for control and prompt, which allows answer-type modification, does not need recursive types, and has ML-like let-polymorphism. We derive the type inference rules from the type structure for the CPS translations for control and prompt recently developed by the above mentioned works. The type system in this paper is a proper extension of that in our previous work [20], which does not allow answer-type modification. We show that our type system enjoys Subject Reduction and Progress properties, and that types are preserved by the CPS translation. The first two properties constitute type soundness, and the third property is necessary for a semantical study.

As an application of our type system, we compare the expressivity of typed control/prompt and that of typed shift/reset. In the type-free setting, they are known to be equally expressive $[7,15,4,6]$. However, there exists a big asymmetry in the complexity of these encodings, and a question remained whether control/prompt is strictly more expressive than shift/reset under an appropriate typed setting (without recursive types). In this paper we answer this question. Namely, we show:

- typed control/prompt can macro-express typed shift/reset, while

- typed shift/reset cannot macro-express typed control/prompt,

where the type system for shift/reset is the most expressive type system by Asai and Kameyama [2]. This result contrasts with the type-free case.

This paper is organized as follows: in Section 2 we briefly explain the control operators for delimited continuations, and in Section 3 we review the functional CPS translation in the literature. The subsequent three sections are original to this paper: in Section 4 we introduce the type systems for the dynamic control operators, and in Section 5 we give several properties for the type systems. In Section 6, we compare the expressive power of typed control/prompt with typed shift/reset. Section 8 concludes.

\footnotetext{
${ }^{1}$ We distinguish (general) recursive types from inductive types in that the former may contain negative occurrences of the type variable being taken the fixed point, for instance, $\mu X$. $(X \rightarrow$ int) where $\mu$ is for the fixed point operator.
} 
The evaluation context captured by control is the whole context since there is no other delimiters. Hence, we obtain a a reversed list as the result of computation.

The operational behavior for each set of control operators can be formalized by reduction rules. Let $v$ and $P$ denote a value and a call-by-value evaluation context such that no delimiter encloses the hole, resp. Then we have the reduction rules as follows:

$$
\begin{aligned}
\text { (For shift } / \text { reset }) & \langle P[\mathcal{S} c . e]\rangle & \rightarrow\langle\text { let } c=\lambda x .\langle P[x]\rangle \text { in } e\rangle \\
(\text { For control } / \text { prompt }) & \#(P[\mathcal{F} \text { c.e }]) & \rightarrow \#(\text { let } c=\lambda x . P[x] \text { in } e)
\end{aligned}
$$

Here $\mathcal{S}$ c.e corresponds to ( $\operatorname{shift~(fn~c~} \Rightarrow e)$ ), and $\langle e\rangle$ to (reset (fn () $\Rightarrow$ $e)$ ) in the ML implementation in the previous subsection. Similarly, \# $e$ is (prompt $(\mathrm{fn}() \Rightarrow e)$ ), and $\mathcal{F}$ c.e is (control (fn c $\Rightarrow e$ )).

Besides the names for control operators, the only difference between them is whether the captured delimited continuation has an extra reset or not: $\lambda x .\langle P[x]\rangle$ for the former, and $\lambda x . P[x]$ for the latter. This small difference in syntax raises a big difference in semantics. Suppose $P$ has other occurrences of shift or control, and the captured delimited continuation is applied to a value $v$ in a then-current continuation $E$ which may have a delimiter that encloses the hole.

- In the former, we evaluate $E[\langle P[v]\rangle]$ in which other occurrences of shift in $P$ will be delimited by this reset (unless they are "escaped" in function closures). Namely, the corresponding delimiter for these shift is determined when the delimited continuation is captured.

- In the latter, we evaluate $E[P[x]]$ in which other occurrences of control ought to be delimited by prompt in $E$. (Note that $P$ does not have any prompt which encloses the hole). Consequently, the corresponding delimiter for these control is determined not when it is captured, but when the delimited continuation is used.

Hence the former is called static, and the latter is called dynamic by analogy with static and dynamic binding in Scheme and Lisp [5]. The static/dynamic nature of control operators has an impact on their implementation. For shift/reset, a delimited continuation can be represented by an ordinary, composable function, which leads to a simple CPS translation $[9,10]$. For control/prompt, we need to keep the captured delimited continuations as they are, until they are actually used, which needs an extra machinery.

\section{A CPS translation for control/prompt}

We regard a CPS translation as the fundamental analysis tool for control operators. For control and prompt, three such translations are known: Shan's [7], Dybvig, Peyton-Jones, and Sabry's [11], and Biernacki, Danvy and Millikin's [6]. In this paper we use (a variant of) the last one since it is the simplest. We could use Shan's one as well. 


$$
\begin{array}{rrr}
v::= & d|x| \lambda x . e & \text { value } \\
e::= & v\left|e_{1} e_{2}\right| \mathcal{F} \text { c.e } \mid \# \text { e } \mid \text { let } x=e_{1} \text { in } e_{2} & \\
& \mid \text { if } e_{1} \text { then } e_{2} \text { else } e_{3} & \\
P::= & {[]|P e| v P} & \\
& \mid \text { let } x=P \text { in } e \mid \text { if } P \text { then } e_{1} \text { else } e_{2} & \text { pure evaluation context } \\
E::= & {[]|E e| v E} & \\
& \mid \text { let } x=E \text { in } e \mid \text { if } E \text { then } e_{1} \text { else } e_{2} \mid \# E & \text { evaluation context }
\end{array}
$$

Fig. 2. Syntax of the language with control/prompt

$$
\begin{aligned}
(\lambda x . e) v & \rightsquigarrow e[v / x] \\
\text { if true then } e_{1} \text { else } e_{2} & \rightsquigarrow e_{1} \\
\text { if false then } e_{1} \text { else } e_{2} & \rightsquigarrow e_{2} \\
\text { let } x=v \text { in } e & \rightsquigarrow e[v / x] \\
\#(P[\mathcal{F} c . e]) & \rightsquigarrow \#(\text { let } c=\lambda x . P[x] \text { in } e) \\
\# v & \rightsquigarrow v
\end{aligned}
$$

Fig. 3. Reduction rules

Figure 2 gives the syntax of our source language where $d$ is constant and $x$ and $c$ are variables. The expression $\mathcal{F}$ c.e is a construct for control in which $c$ is a bound variable. The expression \# $e$ is the one for prompt. Variables are bound by $\lambda$ or $\mathcal{F}$, the set of free variables in $e$ is denoted by $\operatorname{FV}(e)$, and we identify $\alpha$-equivalent expressions. Sequencing $a ; b$ is an abbreviation of $(\lambda x . b) a$ with $x \notin \mathrm{FV}(b)$. In a pure evaluation context $P$, no prompt may enclose its hole [ ], while a (general) evaluation context $E$ allows such occurrences of prompt. Figure 3 gives call-by-value operational semantics to this language where $e[v / x]$ represents the result of capture-avoiding substitution.

Figure 4 defines the CPS translation for this language as a variant of the one given by Biernacki et al. The differences are: (1) they gave a 2CPS translation whch is an iterated translation (its image takes two continuations as its arguments), while we use a more traditional 1CPS translation, and (2) we extend the source language with constant, conditional, and let.

The target language of this translation is a call-by-value lambda calculus with constants, conditional, and let as well as list-manipulating constructs such as Nil, cons (denoted by ::), append (@) and a destructor (case). Note that the translation for let expression is only meaningful for the typed source language (given in the next section), and we have included it in Figure 4 only to save space. For a type-free source language, we can define it as $\llbracket$ let $x=e_{1}$ in $e_{2} \rrbracket=$ $\llbracket\left(\lambda x \cdot e_{2}\right) e_{1} \rrbracket$. 


$$
\begin{aligned}
{[]] } & : \text { SourceTerm } \rightarrow \text { Cont } \rightarrow \text { Trail } \rightarrow \text { TargetValue } \\
{[V] } & =\lambda k t . k(V)^{*} t \\
{\left[e_{1} e_{2}\right] } & =\lambda k t .\left[e_{1}\right]\left(\lambda m_{1} t_{1} .\left[e_{2}\right]\left(\lambda m_{2} t_{2} . m_{1} m_{2} k t_{2}\right) t_{1}\right) t \\
{\left[\text { if } e_{1} \text { then } e_{2} \text { else } e_{3}\right] } & =\lambda k t .\left[e_{1}\right]\left(\lambda m_{1} t_{1} \text {. if } m_{1} \text { then }\left[e_{2}\right] k t_{1} \text { else }\left[e_{3}\right] k t_{1}\right) t \\
{\left[\text { let } x=e_{1} \text { in } e_{2}\right] } & =\lambda k t . \text { let } x=\left(\left[e_{1}\right] \theta_{1} \text { Nil }\right) \text { in }\left[e_{2}\right] k t \\
{[\# e] } & =\lambda k t . k\left([e] \theta_{1} \text { Nil }\right) t \\
{[\mathcal{F} c . e] } & =\lambda k t . \text { let } c=\lambda x k^{\prime} t^{\prime} . k x\left(t @\left(k^{\prime}:: t^{\prime}\right)\right) \text { in }[e] \theta_{1} \text { Nil } \\
(-)^{*} & : \text { SourceValue } \rightarrow \text { TargetValue } \\
(d)^{*} & =d \quad \text { for constant } \\
(x)^{*} & =x \text { for a variable } \\
(\lambda x . e)^{*} & =\lambda x k t .[e] k t \\
\theta_{1} & =\lambda x t . \text { case } t \text { of } \\
& \quad \text { Nil } \Rightarrow x \\
& \mid \quad\left(k_{1}:: t_{1}\right) \Rightarrow k_{1} x t_{1}
\end{aligned}
$$

Fig. 4. 1CPS translation for the language with control and prompt

Let us see the types in Figure 4, although these types should be considered informal, and only for explanation. The types SourceTerm, SourceValue, Cont, TargetValue, are those for terms and values in the source language, and those for continuations and values in the target language, resp.

The type Trail is new to their CPS translation. Recall that, in order to represent the dynamic behavior of control/prompt, we need to keep the delimited continuations until they are used. A trail is a list of delimited continuations to store these continuations. Thus, we may informally define Cont and Trail as follows:

$$
\begin{aligned}
\text { Cont } & =\text { TargetValue } \rightarrow \text { Trail } \rightarrow \text { TargetValue } \\
\text { Trail } & =\text { List }(\text { Cont })
\end{aligned}
$$

This "definition" needs recursive types, which will be examined in the next section.

Next, we look at the term-level translation in Figure 4. For the constructs other than control operators, the translation is the same as the standard (e.g. Plotkin's) translation except that it passes trails without changing them.

The prompt-term $\# e$ initializes its continuation and trail: in its translation $\llbracket \# e \rrbracket, \llbracket e \rrbracket$ is applied to $\theta_{1}$ and the empty trail Nil. The continuation $\theta_{1}$ acts as the identity continuation (the empty evaluation context).

The control-term $\mathcal{F}$ c.e captures a delimited continuation (and initializes the continuation and the trail): it captures the current delimited continuation $k$ for 
a future use of $c$. When $c$ is applied to some value, the captured continuation $k$ is "composed" with the then-current continuation $k^{\prime}$. Rather than simply composing two delimited continuations, we store (in the trail) the list of all the captured delimited continuations except the current one, namely, we extend the then-current trail $t^{\prime}$ to a new trail $t @\left(k^{\prime}:: t^{\prime}\right)$ and use $k$ as the current continuation. Continuations stored in the trail will be used when the current continuation becomes empty (i.e. $\theta_{1}$ ).

Note that we can easily extend our source language and the CPS translation with practical language constructs such as the fixed point operator and primitive functions.

Finally, a CPS translation for a complete program $e$ is defined as $\llbracket e \rrbracket \theta_{1} \mathrm{Nil}$.

\section{Type System}

We introduce a polymorphic type system for control/prompt in this section. Types are an important facility in most programming languages to classify terms and also to ensure a certain kind of safety for computation. We think that, for control/prompt to be used by ordinary programmers, a sound type system is definitely needed.

\subsection{Design of Type System}

Our strategy to construct a type system for control/prompt is basically the same as that for shift/reset [2]: given a term $e$, we infer the most general type of its CPS translation $\llbracket e \rrbracket$, and use this type as the type for $e$. There is, however, two problems in this strategy: (1) the target terms of the CPS translation for control/prompt need recursive types if we assign types, and there is no notion of the most general type in a type system with recursive types, and (2) we need arbitrary many type variables to precisely represent types of the CPS translation.

The first problem already appears if we type the identity (delimited) continuation $\theta_{1}$. Assuming the type of trails is $\operatorname{List}(\tau)$ for some $\tau, \theta_{1}$ must have the type $\alpha \rightarrow \operatorname{List}(X) \rightarrow \alpha$ for some $\alpha$, where $X=\alpha \rightarrow \operatorname{List}(X) \rightarrow \alpha$. Hence we need some sort of recursive types.

The second problem is this: Recall that Cont and Trail are informally defined as Cont $=$ TargetValue $\rightarrow$ Trail $\rightarrow$ TargetValue and Trail $=$ List(Cont) When the source language is typed in some way, the type TargetValue should be instantiated by more specific types, and in general, a trail has the type:

$$
\operatorname{List}\left(\alpha_{1} \rightarrow \operatorname{List}\left(\alpha_{2} \rightarrow \operatorname{List}\left(\ldots \operatorname{List}\left(\alpha_{n} \rightarrow \cdots \rightarrow \beta_{n}\right) \ldots\right) \rightarrow \beta_{2}\right) \rightarrow \beta_{1}\right)
$$

for different $\alpha_{i}$ and $\beta_{i}$. Apparently, we cannot represent these types in a finite manner.

We give a simple solution to these problems based on the observation that most (if not all) examples with control/prompt in the literature can be typed under the following simple restriction: 
- In a trail type, all $\alpha_{i}$ and $\beta_{i}$ is the same type.

This means that any trail must have type $\mu X$.List $(\tau \rightarrow X \rightarrow \tau)$ for some type $\tau$ where $\mu$ is for recursive types. In the sequel, we adopt this restriction, and write Trail $(\tau)$ for this type. Note that the restriction does not constrain the type of continuations, that is, a continuation may have type $\alpha \rightarrow \operatorname{Trail}(\tau) \rightarrow \beta$ for different types $\alpha, \beta$, and $\tau$.

We think that this restriction is not too strong, since, most (if not all) examples with control/prompt in the literature follow this restriction (list-reversing function and several variations of search programs [6]). Also we will see later that the typed control/prompt calculus under this restriction can simulate typed shift/reset calculus, which has many interesting examples.

Under this restriction, the target terms of the CPS translation can be typed in the ordinary type system (with let-polymorphism, but without recursive types), in which the most general type always exists (if typable), and, therefore, the first problem is also solved.

Discussion. An anonymous reviewer has suggested us that, we could exclude the circularity in the trail types by introducing an inductive (not recursive) structure. One way to achieve it is: if we change the definition of the trail type from Trail $=$ List(Cont) to

$$
\operatorname{Trail}(n)=\operatorname{Trail}(0) \mid(\text { Cont } \times \operatorname{Trail}(n-1))
$$

where $n$ denotes the length of the list, then we can avoid the circularity (recursiveness) of the trail type. However, the use of dependent types drastically complicates the type structure of the target calculus (and possibly the corresponding source type system). Moreover, it is not our goal to obtain a strongly normalizing calculus for control/prompt by constraining the source type system artificially. Rather, we took Biernacki et al.'s CPS translation as a good starting point, and based on it, we tried to construct a natural type system which is harmonious with their CPS translation.

\subsection{Definition of Type System}

Now we introduce a type system for control/prompt.

Types and type contexts are defined by:

$$
\begin{array}{rrr}
\alpha, \beta \cdots:: & =b|t| \alpha \rightarrow(\beta, \gamma, \delta / \tau) & \text { monomorphic types } \\
A & ::=\alpha \mid \forall t . A & \text { polymorphic types } \\
\Gamma::=[] \mid \Gamma, x: A & \text { type contexts }
\end{array}
$$

where $b$ is a basic type (including bool), $t$ is a type variable, and $\alpha \rightarrow(\beta, \gamma, \delta / \tau)$ is a function type whose meaning will be made clear later. $\operatorname{FTV}(A)$ denotes the set of free type variables in $A$.

The type system has two forms of judgements, and the first form is:

$$
\Gamma \vdash e: \alpha, \beta, \gamma / \tau
$$




$$
\begin{gathered}
\frac{(x: A \in \Gamma \text { and } \alpha \leq A)}{\Gamma \vdash_{p} x: \alpha} \text { var } \frac{(d \text { is constant of type } b)}{\Gamma \vdash_{p} d: b} \text { const } \\
\frac{\Gamma, x: \alpha \vdash e: \beta, \gamma, \delta / \tau}{\Gamma \vdash_{p} \lambda x . e: \alpha \rightarrow(\beta, \gamma, \delta / \tau)} \text { fun } \frac{\Gamma \vdash_{p} e: \alpha}{\Gamma \vdash e: \alpha, \beta, \beta / \tau} \text { exp } \\
\frac{\Gamma \vdash e_{1}: \alpha \rightarrow(\beta, \gamma, \delta / \tau), \epsilon, \rho / \tau \quad \Gamma \vdash e_{2}: \alpha, \delta, \epsilon / \tau}{\Gamma \vdash e_{1} e_{2}: \beta, \gamma, \rho / \tau} \text { app } \\
\frac{\Gamma \vdash e_{1}: \operatorname{bool}, \delta, \gamma / \tau \quad \Gamma \vdash e_{2}: \alpha, \beta, \delta / \tau \quad \Gamma \vdash e_{3}: \alpha, \beta, \delta / \tau}{\Gamma \vdash \text { if } e_{1} \operatorname{then} e_{2} \text { else } e_{3}: \alpha, \beta, \gamma / \tau} \text { if } \\
\frac{\Gamma \vdash{ }_{p} e_{1}: \rho \quad \Gamma, x: \operatorname{Gen}(\rho ; \Gamma) \vdash e e_{2}: \alpha, \beta, \gamma / \tau}{\Gamma \vdash \operatorname{let} x=e_{1} \text { in } e_{2}: \alpha, \beta, \gamma / \tau} \text { let } \\
\frac{\Gamma \vdash e_{p}: \tau, \tau, \beta / \tau}{\Gamma t \beta} \operatorname{prompt} \frac{\Gamma, c: \alpha \rightarrow(\tau, \tau, \beta / \tau) \vdash e: \rho, \rho, \gamma / \rho}{\Gamma \vdash \mathcal{F} c . e: \alpha, \beta, \gamma / \tau}
\end{gathered}
$$

Fig. 5. Type Inference Rules

where $\Gamma$ is a type context, $\alpha, \beta, \gamma$, and $\tau$ are types, and $e$ is an expression. It means that, under the type context $\Gamma$,e is an expression of type $\alpha$, with the answer type modification $^{2}$ from $\beta$ to $\gamma$, and the trail type is $\tau$. The roles of the additional types in the judgement can be made clearer by CPS translating it:

$$
\begin{gathered}
\Gamma^{*} \vdash \llbracket e \rrbracket: \operatorname{Cont}\left(\alpha^{*}, \beta^{*} / \tau^{*}\right) \rightarrow \operatorname{Trail}\left(\tau^{*}\right) \rightarrow \gamma^{*} \\
\text { with } \\
\operatorname{Trail}\left(\tau^{*}\right)=\mu X . \operatorname{List}\left(\tau^{*} \rightarrow X \rightarrow \tau^{*}\right) \\
\operatorname{Cont}\left(\alpha^{*}, \beta^{*} / \tau^{*}\right)=\alpha^{*} \rightarrow \operatorname{Trail}\left(\tau^{*}\right) \rightarrow \beta^{*}
\end{gathered}
$$

The second form of judgements is:

$$
\Gamma \vdash_{p} e: \alpha
$$

which means $e$ is a pure (effect-free) expression of type $\alpha$. This form is used to introduce let-polymorphism with control effects in a sound manner[2].

Figure 5 gives the type inference rules where $\alpha \leq A$ in the rule (var) means, if $A \equiv \forall t_{1}, \cdots \forall t_{n} . \rho$ for some monomorphic type $\rho$, then $\tau \equiv \rho\left[\sigma_{1}, \cdots, \sigma_{n} / t_{1}, \cdots, t_{n}\right]$ for some monomorphic types $\sigma_{1}, \cdots, \sigma_{n}$. We assume that, a basic type $b$ is associated with each constant $d$. The type $\operatorname{Gen}(\rho ; \Gamma)$ in the rule (let) is defined by $\forall t_{1} . \cdots \forall t_{n} . \rho$ where $\left\{t_{1}, \cdots, t_{n}\right\}=\operatorname{FTV}(\rho)-\operatorname{FTV}(\Gamma)$.

The rule (exp) allows one to switch from the second form to the first form. Since pure expressions are insensitive to continuations and trails, we can introduce arbitrary types for their answer type $(\beta)$ and the trail type $(\tau)$.

\footnotetext{
${ }^{2}$ For the standard CPS translation, we say, the answer type is modified in a computation of $e$ from $\beta$ to $\gamma$, if the CPS transform of $e$ has type $(\alpha \rightarrow \beta) \rightarrow \gamma$. Without control operators for delimited continuations, the types $\beta$ and $\gamma$ are equal. With them, they may be different. See Asai and Kameyama [2] for details.
} 


$$
\begin{gathered}
\frac{\Gamma \vdash e: \alpha, \beta, \gamma / t}{\Gamma \vdash e: \alpha, \beta, \gamma / *} \text { star-intro, if } t \notin \operatorname{FTV}(\Gamma, \alpha, \beta, \gamma) \quad \frac{\Gamma \vdash e: \alpha, \beta, \gamma / *}{\Gamma \vdash e: \alpha, \beta, \gamma / \tau} \text { star-elim } \\
\frac{\Gamma \vdash e: \alpha \rightarrow(\beta, \gamma, \delta / *), \epsilon, \rho / \sigma}{\Gamma \vdash e: \alpha \rightarrow(\beta, \gamma, \delta / \tau), \epsilon, \rho / \sigma} \text { star-fun }
\end{gathered}
$$

Fig. 6. Type Inference Rules for $\lambda_{\text {let }}^{c / p+}$

Other rules are naturally derived from the type for the CPS translation. The rule (var) is standard. In the rule (fun), the function type $\alpha \rightarrow(\beta, \gamma, \delta / \tau)$ extends the ordinary function type $\alpha \rightarrow \beta$ to encapsulate the effect of answer type modification from $\gamma$ to $\delta$ with the trail type $\tau$. The rule (app) reflects this intuition. The rule (let) is for ML-like let-polymorphism. As is well known, we must restrict $e_{1}$ in the expression let $x=e_{1}$ in $e_{2}$ to have a sound type system, and we follow Asai and Kameyama's type system [2] which restricts $e_{1}$ to be a pure term, i.e., either a value or \# $e$.

For the rule (prompt), look at its CPS translation in Figure 4. It is easy to check $\theta_{1}$ must have type $\operatorname{Cont}(\tau, \tau / \tau)$ for some $\tau$, and the return type of $\llbracket e \rrbracket$ is the same as that of $\llbracket \# e \rrbracket$. So by letting this return type $\beta$, we get the rule (prompt). The rule (control) is more complicated. In the CPS translation of $\mathcal{F}$ c.e, a term $\lambda x k^{\prime} t^{\prime} . k x\left(t @\left(k^{\prime}:: t^{\prime}\right)\right)$ is substituted for $c$, which poses constraints that $t$ and $t^{\prime}$ are of the same list type and $k^{\prime}$ is of its member type. Since we restricted all trails to be of type $\operatorname{Trail}(\tau)$ for some $\tau, k^{\prime}$ has to have the type $\operatorname{Cont}(\tau, \tau / \tau)$ and $t$ and $t^{\prime}$ have the type $\operatorname{Trail}(\tau)$. Then we can derive the rule (control).

An example type derivation for a concrete term will be given in a later section.

We call the calculus with this type system $\lambda_{\text {let }}^{c / p}$.

\subsection{Introducing Trail-Polymorphism}

The type system of $\lambda_{\text {let }}^{c / p}$ can type many useful examples with control/prompt. However, it cannot express a certain kind of polymorphism in trails. We occasionally want to express that a function has type $\alpha \rightarrow(\beta, \gamma, \delta / \tau)$ for any $\tau$, i.e., it is insensitive (or polymorphic) to the trail type. To solve this problem with a small cost, i.e., not without introducing impredicative polymorphism, we introduce a limited form of polymorphism, called trail-polymorphism, into $\lambda_{\text {let }}^{c / p}$ as follows.

We add a special type constant "*" to the definition of types, which can appear in a function type as $\alpha \rightarrow(\beta, \gamma, \delta / *)$, or in a judgement as $\Gamma \vdash e$ : $\alpha, \beta, \gamma / *$. Intuitively, $*$ represents a universally quantified type variable.

We add to $\lambda_{\text {let }}^{c / p}$ three new type inference rules listed in Figure 6 , which reflect the intuitive meaning of $*$. We call the extended calculus $\lambda_{\text {let }}^{c / p+}$. Note that the reductions rules are the same as those for $\lambda_{l e t}^{c / p}$. 
We can also introduce impredicative polymorphism in the same manner as that system for shift/reset [2]. Since it is orthogonal to the present type system, we omit the details in this paper.

\section{Properties}

In this section, we state basic properties of $\lambda_{\text {let }}^{c / p}$ and $\lambda_{\text {let }}^{c / p+}$. Due to lack of space, we only state proof sketches in this paper.

The first property, subject reduction, is by far the most important property in a typed calculus.

Theorem 1 (Subject Reduction). If $\Gamma \vdash e_{1}: \alpha, \beta, \gamma / \tau$ is derived and $e_{1} \rightsquigarrow$ $e_{2}$, then $\Gamma \vdash e_{2}: \alpha, \beta, \gamma / \tau$ can be derived.

The theorem is proved by the standard induction on the derivation. For the case of the reduction \# $(P[\mathcal{F}$ c.e $]) \rightsquigarrow \#($ let $c=\lambda x . P[x]$ in $e)$, we decompose it into several smaller reductions as in [3], then the theorem is easy to prove.

The next theorem is the progress property which states the computation of a well typed, closed expression does not get stuck. Here, the word "close" means that the term does not have any free variables and any occurrences of control which are not enclosed by prompt. To ensure the last property, we restrict our attention to an expression in the form \# $e$. A redex is one of the expressions in the lefthand sides of the reduction rules in Figure 3.

Theorem 2 (Progress). If $\vdash \# e: \alpha, \beta, \gamma / \tau$ is derivable, then $\# e$ is in the form $E[r]$ where $E$ is an evaluation context and $r$ is a redex.

Note that, if $e$ is a value $v$, \# $e$ itself is a redex which reduces to $v$. The progress property is proved by the standard case analysis.

These two theorems together constitute the strong type soundness property.

The next theorem states that our type system is compatible with the CPS translation. For this purpose, we define the type structure of the target calculus depending on the source calculus by:

- for $\lambda_{\text {let }}^{c / p}$, the target calculus is (predicatively) polymorphic lambda calculus with conditionals, the trail type $\operatorname{Trail}(\tau)$, and the list type.

- for $\lambda_{\text {let }}^{c / p+}$, the target calculus is impredicatively polymorphic lambda calculus (second order lambda calculus) with conditionals, the trail type $\operatorname{Trail}(\tau)$, and the list type.

We define the CPS translation for types and type contexts by:

$$
\begin{aligned}
\alpha^{*} & =\alpha \quad \text { for basic type and type variable } \\
(\alpha \rightarrow(\beta, \gamma, \delta / \tau))^{*} & =\alpha^{*} \rightarrow\left(\beta^{*} \rightarrow \operatorname{Trail}\left(\tau^{*}\right) \rightarrow \gamma^{*}\right) \rightarrow \operatorname{Trail}\left(\tau^{*}\right) \rightarrow \delta^{*} \\
(\alpha \rightarrow(\beta, \gamma, \delta / *))^{*} & =\alpha^{*} \rightarrow \forall X .\left(\left(\beta^{*} \rightarrow \operatorname{Trail}(X) \rightarrow \gamma^{*}\right) \rightarrow \operatorname{Trail}(X) \rightarrow \delta^{*}\right) \\
{[]^{*} } & =[] \\
\left(\Gamma, x: \forall t_{1} \ldots t_{n} \cdot \alpha\right)^{*} & =\Gamma^{*}, x: \forall t_{1} \ldots t_{n} . \alpha^{*}
\end{aligned}
$$


The third line is for $\lambda_{\text {let }}^{c / p+}$ only. We then state type preservation property as a theorem.

\section{Theorem 3 (Type Preservation for CPS Transformation).}

- $\left(\right.$ For $\lambda_{\text {let }}^{c / p}$ and $\left.\lambda_{\text {let }}^{c / p+}\right)$ If $\Gamma \vdash e: \alpha, \beta, \gamma / \tau$ is derivable for $\tau \neq *$, then $\Gamma^{*} \vdash$ $\llbracket e \rrbracket:\left(\alpha^{*} \rightarrow \operatorname{Trail}\left(\tau^{*}\right) \rightarrow \beta^{*}\right) \rightarrow \operatorname{Trail}\left(\tau^{*}\right) \rightarrow \gamma^{*}$ is derivable in the target calculus.

- $\left(\right.$ For $\left.\lambda_{\text {let }}^{c / p+}\right)$ If $\Gamma \vdash e: \alpha, \beta, \gamma / *$ is derivable, then $\Gamma^{*} \vdash \llbracket e \rrbracket:\left(\alpha^{*} \rightarrow\right.$ $\left.\operatorname{Trail}(\tau) \rightarrow \beta^{*}\right) \rightarrow \operatorname{Trai} l(\tau) \rightarrow \gamma^{*}$ is derivable for any $\tau$ in the target calculus.

In this paper, we do not state the property that equality is preserved by CPS translation, since it is independent to our type system, and in order to state the property as a theorem, we need to develop a sophisticated equality theory in the target language. For the latter, we need the following property for an arbitrary pure evaluation context $P$ :

$$
\lambda x k_{1} t_{1} \cdot \llbracket P[x] \rrbracket \theta_{1}\left(k_{1}:: t_{1}\right)=\lambda x k_{1} t_{1} \cdot \llbracket P[x] \rrbracket k_{1} t_{1}
$$

which is about the inductive nature of trails (lists). Since $t_{1}$ in this equation is a bound variable, we need to elaborate an inductive theory.

\section{Encoding Shift/Reset by Control/Prompt}

In type-free setting, shift/reset can be macro-defined by control/prompt [4], and we show in this section that it also holds for the typed setting here. We adopt the calculus $\lambda_{\text {let }}^{s / r}$ in [2] for shift/reset, since it is the most liberal type system which allows answer-type modification and let-polymorphism. Its type inference rules are listed in the appendix.

We define a translation from $\lambda_{\text {let }}^{s / r}$ to $\lambda_{\text {let }}^{c / p+}$ as follows:

$$
\begin{aligned}
& \text { Types } \quad \overline{(\alpha / \gamma \rightarrow \beta / \delta)}=\bar{\alpha} \rightarrow(\bar{\beta}, \bar{\gamma}, \bar{\delta} / *) \\
& \text { Expressions } \quad \overline{\mathcal{S} c . e}=\mathcal{F} c^{\prime} \text {.let } c=\lambda x . \#\left(c^{\prime} x\right) \text { in } \bar{e} \\
& \overline{\langle e\rangle}=\# \bar{e} \\
& \text { Type Contexts } \quad \overline{\Gamma, x: \forall t_{1} \ldots t_{n} \cdot \alpha}=\bar{\Gamma}, x: \forall t_{1} \ldots t_{n} \cdot \bar{\alpha}
\end{aligned}
$$

For other constructs, the translation is homomorphic. This translation preserves types and reduction.

\section{Theorem 4.}

- If $\Gamma ; \beta \vdash e: \alpha ; \gamma$ is derivable in $\lambda_{\text {let }}^{s / r}, \bar{\Gamma} \vdash \bar{e}: \bar{\alpha}, \bar{\beta}, \bar{\gamma} / *$ is derivable in $\lambda_{\text {let }}^{c / p+}$.

- If $\Gamma ; \beta \vdash e_{1}: \alpha ; \gamma$ is derivable and $e_{1} \rightsquigarrow e_{2}$ in $\lambda_{\text {let }}^{s / r}, \overline{e_{1}} \rightsquigarrow * \overline{e_{2}}$ in $\lambda_{\text {let }}^{c / p+}$. 
Proof. We prove this theorem by induction on the derivation of $\Gamma ; \beta \vdash e: \alpha ; \gamma$. We list two key cases here.

(Reset) Suppose we have a derivation in $\lambda_{\text {let }}^{s / r}$ :

$$
\frac{\Gamma ; \alpha \vdash e: \alpha ; \beta}{\Gamma ; \gamma \vdash\langle e\rangle: \beta ; \gamma} \text { reset }
$$

By induction hypothesis, we can derive $\bar{\Gamma} \vdash \bar{e}: \bar{\alpha}, \bar{\alpha}, \bar{\beta} / *$. Then we can derive:

$$
\begin{gathered}
\frac{\bar{\Gamma} \vdash \bar{e}: \bar{\alpha}, \bar{\alpha}, \bar{\beta} / *}{\bar{\Gamma} \vdash \bar{e}: \bar{\alpha}, \bar{\alpha}, \bar{\beta} / \bar{\alpha}} \\
\overline{\bar{\Gamma} \vdash} \vdash_{p} \# \bar{e}: \bar{\beta} \\
\bar{\Gamma} \vdash \overline{\langle e\rangle}=\# \bar{e}: \bar{\beta}, \bar{\gamma}, \bar{\gamma} / *
\end{gathered}
$$

(Shift) Suppose we have a derivation in $\lambda_{\text {let }}^{s / r}$ :

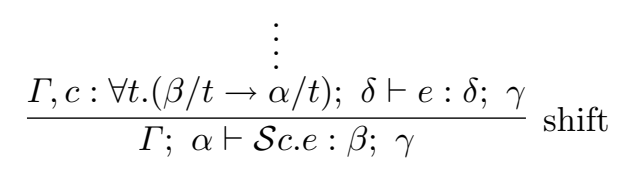

Let $\Delta_{1}=\bar{\Gamma}, c: \forall t .(\bar{\beta} \rightarrow(\bar{\alpha}, t, t / *))$. By induction hypothesis, we have a derivation for $\Delta_{1} \vdash e: \bar{\delta}, \bar{\delta}, \bar{\gamma} / *$ in $\lambda_{\text {let }}^{c / p+}$. Let $\Delta_{2}=\bar{\Gamma}, c^{\prime}: \bar{\beta} \rightarrow(s, s, \bar{\alpha} / s)$ where $s$ is a fresh type variable, then we can derive:

$$
\begin{aligned}
& \frac{\Delta_{2}, x: \bar{\beta} \vdash c^{\prime} x: s, s, \bar{\alpha} / s}{\Delta_{2}, x: \bar{\beta} \vdash{ }_{p} \#\left(c^{\prime} x\right): \bar{\alpha}} \\
& \overline{\Delta_{2}, x: \bar{\beta} \vdash \#\left(c^{\prime} x\right): \bar{\alpha}, t, t / *} \\
& \overline{\Delta_{2} \vdash_{p} \lambda x . \#\left(c^{\prime} x\right): \bar{\beta} \rightarrow(\bar{\alpha}, t, t / *)} \quad \Delta_{1} \vdash e: \bar{\delta}, \bar{\delta}, \bar{\gamma} / * \\
& \overline{\Delta_{2} \vdash_{p} \lambda x . \#\left(c^{\prime} x\right): \bar{\beta} \rightarrow(\bar{\alpha}, t, t / *)} \quad \overline{\Delta_{1} \vdash e: \bar{\delta}, \bar{\delta}, \bar{\gamma} / \bar{\delta}} \\
& \frac{\Delta_{2} \vdash \text { let } c=\lambda x . \#\left(c^{\prime} x\right) \text { in } \bar{e}: \bar{\delta}, \bar{\delta}, \bar{\gamma} / \bar{\delta}}{\bar{\Gamma} \vdash \mathcal{F} c^{\prime} \cdot \text { let } c=\lambda x \cdot \#\left(c^{\prime} x\right) \text { in } \bar{e}: \bar{\beta}, \bar{\alpha}, \bar{\gamma} / s}
\end{aligned}
$$

Since $s \notin \operatorname{FTV}(\bar{\Gamma}, \bar{\alpha}, \bar{\beta}, \bar{\gamma})$, we can derive $\bar{\Gamma} \vdash \overline{\mathcal{S} c . e}: \bar{\beta}, \bar{\alpha}, \bar{\gamma} / *$.

We can also show that, if $e \rightsquigarrow e^{\prime}$ in $\lambda_{\text {let }}^{s / r}$, then $\bar{e} \rightsquigarrow * \bar{e}^{\prime}$ in $\lambda_{\text {let }}^{c / p+}$, whose key case is proved as follows:

$$
\begin{aligned}
\overline{\langle P[\mathcal{S} c . e]\rangle} & =\#\left(\bar{P}\left[\mathcal{F} c^{\prime} \cdot \text { let } c=\lambda x \cdot \# c^{\prime} x \text { in } \bar{e}\right]\right) \\
& \rightsquigarrow \#\left(\text { let } c^{\prime}=\lambda y \cdot \bar{P}[y] \text { in let } c=\lambda x . \# c^{\prime} x \text { in } \bar{e}\right) \\
& \rightsquigarrow \#(\text { let } c=\lambda y \cdot \#(\lambda y \cdot \bar{P}[x]) x \text { in } \bar{e}) \\
& \rightsquigarrow \#(\text { let } c=\lambda x \cdot \# \bar{P}[x] \text { in } \bar{e}) \\
& =\overline{\langle\text { let } c=\lambda x \cdot\langle P[x]\rangle \text { in } e\rangle}
\end{aligned}
$$

We remark that this proof does not work for $\lambda_{\text {let }}^{c / p}$. 


\section{Typed Control/Prompt is Strictly More Expressive Than Shift/Reset}

In the type-free setting, we can encode control/prompt in terms of shift/reset $[7,15,6]$. In the typed setting, it is not the case, as we will prove in this section. Since $\lambda_{\text {let }}^{s / r}$ is strongly normalizing [2], it is sufficient to construct a typable expression in $\lambda_{\text {let }}^{c / p+}$ (or $\lambda_{\text {let }}^{c / p}$ ) whose computation is not terminating.

Let $\alpha$ and $\bullet$, resp. be a type and its inhabitant, resp., for instance, $\alpha=$ bool and $\bullet=$ true. Let $\Gamma$ be the type context $c: \alpha \rightarrow(\alpha, \alpha, \alpha / \alpha)$, and recall $e_{1} ; e_{2}$ is an abbreviation of $\left(\lambda x . e_{2}\right) e_{1}$ for $x \notin \mathrm{FV}\left(e_{2}\right)$. We can type the expression $\#(\mathcal{F} c .(c \bullet ; c \bullet) ; \mathcal{F} c .(c \bullet ; c \bullet))$ in $\lambda_{\text {let }}^{c / p}$ as follows:

$$
\begin{gathered}
\frac{\overline{\Gamma \vdash c: \alpha \rightarrow(\alpha, \alpha, \alpha / \alpha), \alpha, \alpha / \alpha} \overline{\Gamma \vdash \bullet: \alpha}}{\frac{\Gamma \vdash c \bullet: \alpha, \alpha, \alpha / \alpha}{\Gamma \vdash c \bullet ; c \bullet: \alpha, \alpha, \alpha / \alpha}} \\
\frac{\frac{\vdash_{p} \mathcal{F} c .(c \bullet ; c \bullet): \alpha}{\vdash \mathcal{F} c .(c \bullet ; c \bullet) ; \mathcal{F} c .(c \bullet ; c \bullet): \alpha, \alpha, \alpha / \alpha}}{\vdash \#(\mathcal{F} c .(c \bullet ; c \bullet) ; \mathcal{F} c .(c \bullet ; c \bullet)): \alpha, \beta, \beta / \tau}
\end{gathered}
$$

The computation of this term does not terminate:

$$
\begin{aligned}
& \#(\mathcal{F} c .(c \bullet ; c \bullet) ; \mathcal{F} c .(c \bullet ; c \bullet)) \\
& \rightsquigarrow \#(\text { let } c=\lambda u .(u ; \mathcal{F} c .(c \bullet ; c \bullet)) \text { in }(c \bullet ; c \bullet)) \\
& \rightsquigarrow^{*} \#(\mathcal{F} c .(c \bullet ; c \bullet) ;(\lambda u .(u ; \# \mathcal{F} c .(c \bullet ; c \bullet)) \bullet) \\
& \rightsquigarrow{ }^{*} \#\left(\mathcal{F} c .(c \bullet ; c \bullet) ;\left(\lambda u^{\prime} .\left(u^{\prime} ;(\lambda u .(u ; \# \mathcal{F} c .(c \bullet ; c \bullet)))\right)\right) \bullet\right) \\
& \rightsquigarrow{ }^{*} \ldots
\end{aligned}
$$

Since this example does not use answer-type modification or polymorphism, it can be typed in a more restricted type system such as our previous one [20].

We can go a step further. After submission of this paper, Kiselyov [16] and the second author (Yonezawa) have independently constructed fixed point combinators in call-by-value:

$$
\begin{aligned}
(\text { Kiselyov }) & \mathrm{Y}_{1}=\lambda f . \#\left(Z_{f} ; Z_{f}\right) & \text { where } Z_{f}=\mathcal{F} c . f(\lambda x . \#(c \bullet ; \bullet \bullet) x) \\
(\text { Yonezawa }) & \mathrm{Y}_{2}=\lambda f . \#\left(X_{f} ; X_{f}\right) & \text { where } X_{f}=\mathcal{F} c . \lambda x .(f \#(c \bullet ; \bullet \bullet)) x
\end{aligned}
$$

Both $\mathrm{Y}_{1}$ and $\mathrm{Y}_{2}$ are typable in $\lambda_{\text {let }}^{c / p}$, and satisfy $\mathrm{Y}_{1} f x=f\left(\lambda x . \mathrm{Y}_{1} f x\right) x$, and $\mathrm{Y}_{2} f x=f\left(\lambda x . f\left(\mathrm{Y}_{2} f\right) x\right) x$. Therefore, they can serve as fixed point combinators. The former satisfies a simpler equation, while $\mathrm{Y}_{1} e$ may not terminate for some term $e$, but $\mathrm{Y}_{2} e$ always terminates.

\section{Conclusion}

We have introduced a polymorphic type system for control/prompt, which allows answer type modification and does not need recursive types. We have shown 
that our calculus enjoys type soundness and is compatible with the CPS translation, and that typed control/prompt is strictly more powerful than typed shift/reset in the absence of recursive types. Although we cannot claim that our type system is *the* only type system for control/prompt, we believe that ours can be a good starting point to study the type structure of these control operators.

Based on this work, Kiselyov and the second author have successfully shown that $\lambda_{\text {let }}^{c / p}$ is Turing-complete if we extend the calculus with integers and primitive functions. The situation is similar to the exception mechanism in ML, for which Lillibridge [17] has proved that typed (unchecked) exception can simulate all type-free lambda terms, and therefore can represent all Turing-computable functions. Thielecke also compared the expressive powers of several control operators using a different technique [19].

In this paper we have been concentrating on the foundational aspect of control/prompt in this paper, and the practical aspect of our type systems is unstudied. In particular, more application programs other than those by Biernacki, Danvy, Millikin's are called for, but it is left for future work.

Acknowledgements: We thank Kenichi Asai, Dariusz Biernacki, Olivier Danvy, and Chung-chieh Shan for their insights. Special thanks go to Oleg Kiselyov and anonymous reviewers for valuable comments. This work was partly supported by JSPS Grant-in-Aid for Scientific Research (C) 16500004.

\section{References}

1. K. Asai. On Typing Delimited Continuations: Three New Solutions to the Printf Problem. Technical Report OCHA-IS 07-1, Department of Information Science, Ochanomizu University, September 2007.

2. K. Asai and Y. Kameyama. Polymorphic Delimited Continuations. In Proc. Asian Programming Languages and Systems, LNCS 4807, pages 239-254, Nov-Dec 2007.

3. K. Asai and Y. Kameyama. Polymorphic Delimited Continuations. Technical Report CS-TR-07-10, Dept. of Computer Science, University of Tsukuba, Sep 2007.

4. D. Biernacki and O. Danvy. A Simple Proof of a Folklore Theorem about Delimited Control. J. Funct. Program., 16(3):269-280, 2006.

5. D. Biernacki, O. Danvy, and C. c. Shan. On the Static and Dynamic Extents of Delimited Continuations. Science of Computer Programming, 60(3):274-297, 2006.

6. D. Biernacki, O. Danvy, and K. Millikin. A Dynamic Continuation-Passing Style for Dynamic Delimited Continuations. TOPLAS, to appear.

7. C. c. Shan. Shift to control. In Proc. Workshop on Scheme and Functional Programming, pages 99-107, 2004.

8. O. Danvy. Functional Unparsing. J. Funct. Program., 8(6):621-625, 1998.

9. O. Danvy and A. Filinski. Abstracting Control. In Proc. 1990 ACM Conference on Lisp and Functional Programming, pages 151-160, 1990.

10. O. Danvy and A. Filinski. Representing Control: a Study of the CPS Transformation. Mathematical Structures in Computer Science, 2(4):361-391, 1992.

11. R. K. Dybvig, S. Peyton Jones, and A. Sabry. A Monadic Framework for Delimited Continuations. J. Funct. Program., to appear.

12. M. Felleisen. The Theory and Practice of First-Class Prompts. In Proc. 15th Symposium on Principles of Programming Languages, pages 180-190, 1988. 
13. A. Filinski. Representing Monads. In POPL, pages 446-457, 1994.

14. Y. Kameyama and M. Hasegawa. A sound and complete axiomatization for delimited continuations. In ICFP, pages 177-188, 2003.

15. O. Kiselyov. How to remove dynamic prompt: Static and dynamic delimited continuation operators are equally expressive. Technical Report 611, Computer Science Department, Indiana University, March 2005.

16. O. Kiselyov. Fixpoint combinator from typed prompt/control. 2007. http://okmij .org/ftp/Computation/Continuations.html.

17. M. Lillibridge. Unchecked Exceptions Can Be Strictly More Powerful Than Call/CC. Higher-Order and Symbolic Computation, 12(1):75-104, 1999.

18. C. Strachey and C. P. Wadsworth. Continuations: A mathematical semantics for handling full jumps. Technical Monograph PRG-11, Oxford Univ. Comput. Lab., Oxford, England, 1974. Reprinted in Higher-Order and Symbolic Computation 13(1/2):135-152, 2000.

19. H. Thielecke. On Exceptions Versus Continuations in the Presence of State. In ESOP, pages 397-411, 2000.

20. T. Yonezawa and Y. Kameyama. A Type System for Dynamic Delimited Continuations. IPSJ Transactions on Programming, Information Processing Society of Japan, to appear.

\section{A Polymorphic Type System for shift/reset}

We define the type system for $\lambda_{\text {let }}^{s / r}$ in [2] except the fixed point operator. Types and type contexts are defined by:

$$
\begin{array}{rrr}
\alpha, \beta \cdots:: & =b|t|(\alpha / \beta \rightarrow \gamma / \delta) & \text { monomorphic types } \\
A & ::=\alpha \mid \forall t . A & \text { polymorphic types } \\
\Gamma: & :=[] \mid \Gamma, x: A & \text { type contexts }
\end{array}
$$

where the function type $(\alpha / \beta \rightarrow \gamma / \delta)$ corresponds to $\alpha \rightarrow(\gamma, \beta, \delta / *)$ in $\lambda_{\text {let }}^{c / p+}$. Judgements in $\lambda_{\text {let }}^{s / r}$ are either $\Gamma$; $\alpha \vdash e: \beta$; $\gamma$ or $\Gamma \vdash_{p} e: \beta$. The former corresponds to $\Gamma \vdash e: \beta, \alpha, \gamma / *$ in $\lambda_{\text {let }}^{c / p+}$. Finally, Figure 7 gives several important type inference rules of $\lambda_{\text {let }}^{s / r}$.

$$
\begin{gathered}
\frac{\Gamma, x: \sigma ; \alpha \vdash e: \tau ; \beta}{\Gamma \vdash_{p} \lambda x \cdot e:(\sigma / \alpha \rightarrow \tau / \beta)} \text { fun } \\
\frac{\Gamma, k: \forall t .(\tau / t \rightarrow \alpha / t) ; \sigma \vdash e: \sigma ; \beta}{\Gamma ; \alpha \vdash \mathcal{S k} . e: \tau ; \beta} \operatorname{shift} \quad \frac{\Gamma ; \sigma \vdash e: \sigma ; \tau}{\Gamma \vdash_{p}\langle e\rangle: \tau} \text { reset }
\end{gathered}
$$

Fig. 7. Type Inference Rules of $\lambda_{\text {let }}^{s / r}$. 